\title{
ON PORTFOLIO OPTIMIZATION: ARE THERE FINANCIAL GAINS OF USING ALTERNATIVE COVARIANCE METHODS?
}

\author{
Ahsen Saghir $^{1 *}$, Syed Muhammad Ali Tirmizi ${ }^{2}$, Ch Kamran Mahmood ${ }^{3}$, Nauman Iqbal Mirza ${ }^{4}$, Naeem Khan ${ }^{5}$ \\ $1^{*}, 3,4,5$ Department of Business Administration, Foundation University Rawalpindi Campus, Pakistan; ${ }^{2}$ Assistant \\ Professor, Department of Business Administration, Foundation University Rawalpindi Campus, Pakistan. \\ Email: *ahsensaghir@gmail.com
}

Article History: Received on $17^{\text {th }}$ June 2021, Revised on $22^{\text {nd }}$ June 2021, Published on $23^{\text {rd }}$ June 2021

\begin{abstract}
Purpose: The study evaluates the performance of alternative variance-covariance estimators as a fundamental ingredient to portfolio optimization.

Methodology: The study estimates eleven covariance matrices on the data of Pakistan stock exchange's non-financial sector firms covering the period from July 2006 to June 2020. The accuracy and efficiency of covariance estimators are assessed through two evaluation parameters: root mean square error and minimum variance portfolios (risk behavior).
\end{abstract}

Main findings: Empirical findings based on evaluation parameters suggest that more complex covariance estimators in the equity market of Pakistan yield no additional financial gains than the equally weighted portfolio of estimators.

\begin{abstract}
Application of the study: As the estimation of the variance-covariance matrix is one of the essential elements of portfolio construction, this study guides investor(s) on selecting an appropriate covariance estimator among eleven estimators endorsed by literature.
\end{abstract}

Novelty/ originality of the study: Based on detailed analysis, the study documents that investor(s) of the Pakistan stock exchange cannot gain any additional benefit from more complex and tricky methods of variance-covariance estimators compared to a portfolio of estimators for the non-financial sector. Investors are advised to consider the equally weighted portfolio of estimators when formulating their investment strategy.

Keywords: Variance Covariance Estimators, Portfolio Optimization, RMSE, PSE.

\section{INTRODUCTION}

The idea of diversification and portfolio optimization is instrumental in interpreting financial markets and investment decision-making. In this context, the publication; theory of portfolio selection by Harry Markowitz in 1952 is considered a significant breakthrough. His theory, commonly known as modern portfolio theory (MPT), provides the answer to a fundamental question of any investor: How to distribute funds into different investment avenues? By using the statistical procedure, Markowitz (1952) quantified risk and the return of securities in the form of standard deviation and expected return, respectively. Markowitz also suggested that any security risk and return should be considered collectively, and the investment decision should solely be based on risk and return's tradeoff. The groundbreaking work of Markowitz on mean-variance optimization is greatly valued in the literature of finance. The work of Markowitz is still in practice; researchers and portfolio managers are actively considering the traditional version for the construction of financial portfolios (Jagannathan \& Ma, 2003; Tu \& Zhou, 2011).

The mean-variance by Markowitz (1952) remained under fire at multiple fronts. Michaud (1989) labels the meanvariance optimization as "enigma". In his view, the Markowitz efficient frontier method of investment diversification suggests false, irrelevant, and worthless weights for the placement of assets, says the resulting portfolios are "counterintuitive". Stepping ahead, Michaud (1989) termed the whole framework as an "estimation error maximizer". Disatnik and Benninga (2007) question the reliability of estimates and argue that this method produces doubtful returns. Ledoit and Wolf (2003) term estimation of variance-covariance matrix, a tiring part of the method. Best and Grauer (1991) and Chopra and Ziemba (1993) argue that the method of Markowitz is input centred and raised concern over the robustness of the optimization strategy. Chow et al. (1995) discard the mean-variance approach and argue that any investor's utility is not just based on risk and expected return function. Literature is evident that researchers and portfolio engineers failed to provide a systematic, flawless approach for building an optimized portfolio (Ly, 2019; Zakamulin, 2017). Following the positive side of the mean-variance optimization, other researchers applied the fundamental idea and enhanced the original Markowitz's optimization model. Konno and Yamazaki (1991) applied the mean absolute deviation technique for the optimization of the portfolio as a solution to the portfolio problem. Levy and Markowitz (1979) introduced the application of linear programming, on the same concept Tamiz and Jones (1996) applied goal programming for portfolio optimization.

The review of the literature regarding portfolio optimization deals with two aspects; theoretical aspect and implementation aspect. The theoretical aspect discusses the assumption of portfolio optimization and re-evaluates these assumptions, whereas the implementation aspect deals with two necessary elements required for building and implementing the portfolio model. From an investor's point of view, there are two fundamental elements as input to portfolio construction. The first is the estimation of return vectors, and the second is the estimation of the covariance matrix. This research mainly focuses on estimating the second element required to build a portfolio, using different 
variance-covariance approaches endorsed by literature. The best-suited variance-covariance approach can then be recommended to the investor of the Pakistan stock exchange for the construction of an optimized portfolio.

The following section of the literature review sheds light on the estimation techniques of variance-covariance matrix used around the globe for the construction of portfolios.

\section{LITERATURE REVIEW}

Financial literature reports numerous efforts of researchers for a reliable solution to the problem of portfolio optimization. Over time, they proposed a broad range of methods, rules, theories, strategies, and frameworks, etc., to achieve an optimal solution for the placement of investors' wealth. The below segment of the literature mainly reviews variance-covariance estimators that serve as an essential component for constructing an optimized portfolio.

The sample variance-covariance matrix procedure depends on historical covariance and estimates pairwise variancecovariance of the sample asset category. This pairwise covariance estimation is vulnerable to errors, mainly when the underlying asset groups are larger than the sample asset groups (Hwang et al., 2018; Pafka \& Kondor, 2004). Sharpe $\underline{(1963)}$ strengthens the sample variance-covariance matrix approach by proposing a more robust covariance method based on a single market factor. Other researchers also came forward and tried to improve the performance of the sample covariance technique. Other than a single common factor, King (1966) discusses several others elements. Vasicek (1973) optimize the covariance estimator's efficiency by using a mean-reverting bias and changing the beta variation, respectively.

According to Husnain et al. (2016), the typical method for estimating the covariance matrix is susceptible to errors, maybe due to estimation or specification errors. Non-theory-based or predictive measures, such as principal component analysis (PCA), have often been used in the literature to identify variables related to sample covariance. For asset placement, Elton and Gruber (1973) propose the use of average correlation-based variance-covariance estimators. Nontheory-based or statistic-based diversification measures outperform complex theory-based optimization techniques due to the volatility of computational estimators and estimation problems (DeMiguel et al., 2007). The decision theory of statistics directs to an optimized spot between the specification and estimation problem. For optimization between these two, researchers look to this basic norm of statistics. According to Stein (1956), the optimized spot can be determined by estimating the weighted average of both estimators. The work on the efficacy of shrinkage-based estimation and portfolios of estimators using empirical data to estimate the covariance matrix can be attributed to (Bengtsson \& Holst, 2002; Ledoit \& Wolf, 2003, 2004).

In contrast to the traditional sample variance-covariance method and the single index variance-covariance approach, Ledoit and Wolf $(2003,2004)$ suggest the Bayesian shrinkage strategy of portfolio optimization. In the case of sample covariance, this approach eliminates issues, including all measurement errors as well as all specification errors. This method produces a matrix that is smaller than the traditional sample matrix and is called shrunk matrix. All offdiagonal components (covariances) are shrunk while the diagonal components remain unchanged. Ledoit and Wolf (2003, 2004) Ledoit and Wolf (2004) successfully shrink traditional sample covariance matrices into the constant correlation matrix. However, Jagannathan and Ma (2003) criticized and challenged the shrinkage variance-covariance technique.

As per the review of literature, it is found that a consensus could not be developed among researchers on the use of covariance estimation methods in the world and in the stock market of developing counties. The current research undertakes the estimation and evaluation of eleven variance-covariance estimation methods using the Pakistan stock exchange's non-financial market data. For the evaluation of covariance estimators, the literature linked with portfolio optimization suggests two evaluation parameters. First, the root mean square error (RMSE) to comment on the performance of covariance estimator second, is minimum variance portfolio (MVP). The MPV looks at-risk behaviour of each estimator and guides the efficiency of variance-covariance estimators.

The rest of the article is structured as follows: the third section explains datasets, methods used, and evaluation parameters of the variance-covariance estimators. The fourth section presents results and interpretations. And, the fifth section presents major findings and the conclusion of the study.

\section{DATA AND METHODOLOGY}

The study sample consists of the share prices of non-financial firms listed at the PSX. The weekly data of equity prices is collected from the website of the Pakistan stock exchange. A relatively large dataset spanning over 14 years is considered for analysis, ranging from $01 / 07 / 2006$ to 30/06/2020. The dataset is further divided into two subsamples from $01 / 07 / 2006$ to $30 / 06 / 2013$ and $01 / 07 / 2013$ to $30 / 06 / 2020$. The first subsample is used to calculate variance-covariance estimators, while the second subsample is used to check the ex-post efficiency of the estimators. Indices for 22 sectors of PSX are formulated based on the equally weighted method.

Under the assumption of continuous compounding, return are calculated for each asset category as: $\left(R_{i, t}\right)=\ln \left(P_{t} / P_{t-1}\right)$. Here, $\ln$ denotes to natural $\log , P_{t}$ to recent price, and $P_{t-1}$ to the preceding price of asset category. The section below 
provides detail of various approaches to variance-covariance matrices concerning their origin, working structure, and usefulness.

\section{Detail of variance-covariance estimators}

The literature documents the use of alternative approaches of variance-covariance for the construction of portfolios. Table 1 presents a list of approaches endorsed by literature with their symbols for the construction of portfolios.

The study estimates and evaluates eleven approaches of variance-covariance under four different groups. The approaches are grouped under four categories: Traditional approaches, Factor Model, portfolio of estimators, and shrinkage approaches of variance-covariance estimators. Traditional approaches include; the sample variance-covariance model and the constant correlation variance-covariance model. Factor model contains; the single index method to variancecovariance. Equally weighted methods of variance-covariance proposed by Ledoit and Wolf (2003) are grouped under the head of the portfolio of estimators, while the shrinkage approaches of variance-covariance include; shrinkage to diagonal, shrinkage to single index, and shrinkage to the constant correlation matrix.

Table 1: Variance-covariance estimators with their symbols

\begin{tabular}{lll}
\hline Sr. & Variance-Covariance Estimators & Symbols \\
\hline & Traditional Methods & DC \\
\hline $\mathbf{1}$ & The diagonal var. covariance matrix & SC \\
\hline $\mathbf{2}$ & The sample var. covariance model & CC \\
\hline & The constant correlation var. covariance model & SI \\
\hline $\mathbf{4}$ & The single index var. covariance model & \\
\hline & Portfolio of Estimators & P1 \\
\hline $\mathbf{5}$ & The portfolio based on sample \& diagonal matrix & P2 \\
\hline $\mathbf{6}$ & The portfolio based on sample \& single index matrix & P3 \\
\hline $\mathbf{7}$ & The portfolio based on sample \& constant correlation covar. matrix \\
\hline $\mathbf{8}$ & The portfolio is based on sample, constant corr. \& single index matrix & P4 \\
\hline $\mathbf{9}$ & The portfolio based on the sample, single index \& overall mean matrix & P5 \\
\hline & Shrinkage Approaches & SD \\
\hline $\mathbf{1 0}$ & The shrinkage aimed at a diagonal point & SSI \\
\hline $\mathbf{1 1}$ & The shrinkage aimed at a single index point & SCC \\
\hline $\mathbf{1 2}$ & The shrinkage aimed at a constant correlation point & \\
\hline
\end{tabular}

The diagonal method of variance-covariance is a basic component of variance-covariance matrix estimation. Elton and Gruber (1973) suggest the use of a sample covariance estimator; this method uses past values; however, it is based on a flawed system in comparison to other covariance estimators. Likewise, Sharpe (1963) considers a systematic approach for risk factors and an estimated matrix of covariance. Researchers criticized this approach as it was based on the single factor of risk and specification fault. Despite the criticism, it proved its worth and performed much better than the sample covariance method, but this approach had a built-in fault of producing specification errors. Ledoit and Wolf (2003, 2004) Ledoit and Wolf (2004) propose an optimal shrinkage method. This method shrinks two covariance matrices toward a target matrix to get one estimator of covariance. Jagannathan and Ma (2003) propose a relatively simple approach of equally-weighted average. This approach estimates the covariance of two or more covariance methods using their weighted average.

\section{The variance-covariance matrix estimation}

For all categories of involved assets, this square matrix consists of both variance and covariances whose diagonal values consist of variance for each asset category, whereas its off-diagonal values consist of covariance for possible categories of asset pairs. To put it another way, variance refers to the squared mean difference while covariance represents the motion of two asset categories.

The variance-covariance matrix can be expressed mathematically as: 


$$
\sum=\left[\begin{array}{cccc}
\sum i_{1}^{2} / j & \sum i_{1} i_{2} / j & & \sum i_{1} i_{i} / j \\
\sum i_{2} i_{1} / j & \sum i_{2}^{2} / j & \cdots & \sum i_{2} i_{k} / j \\
\cdots & \cdots & \cdots & \cdots \\
\sum i_{k} i_{1} / j & \sum i_{i} i_{2} / j & & \sum i_{k}^{2} / j
\end{array}\right]
$$

Where; $\sum$ denotes to the variance-covariance matrix $(k \times k) . j$ denotes to points of data for each asset category while $i_{1}$ denotes to average deviation and $i_{1}^{2} / j$ denotes to the covariance of asset category of $n$ and $m$.

\section{The sample variance-covariance matrix}

For $\left(\mathrm{s} \in \mathrm{t}^{\mathrm{m}}\right)$ vector, the sample variance is presented with $\sigma^{2}$ whereas sample average is presented with $\hat{\mathrm{z}}$, then:

$$
\begin{gathered}
\hat{s}=\frac{1}{j} *\left(s_{1}+s_{2}+s_{3}+\cdots+s_{j}\right) \\
\sigma 2=\frac{1}{j} *\left(\left(s_{1}-\widehat{s}\right)^{2}+\left(s_{2}-\widehat{s}\right)^{2}+\cdots+\left(s_{j}-\widehat{s}\right)^{2}\right)
\end{gathered}
$$

Let, $Z=\left[z_{1}+z_{2}+z_{3}+\cdots+z_{n}\right] \in P^{i * j}$, in the preceding expression, every column $z_{k}$ presents a figure in $P^{j}$. Here, variance values are captured from the projection of the data using $\rho \in P^{i}$, such that:

$$
s=\left(\rho^{C} y_{1}+\rho^{C} y_{2}+\rho^{C} y_{3}+\cdots \rho^{C} y_{j}\right)=\rho^{C} Z \in P^{j}
$$

Whereas the corresponding mean of sample and variance are:

$$
\sigma^{2} *(\rho)=\frac{1}{j} \sum_{l=1}^{j}\left(\rho^{C} z_{l}-\rho^{C} \hat{z}\right)^{2}, \hat{s}=\rho^{C} \hat{z}
$$

In preceding expression, mean of sample for $\hat{z}=\frac{1}{j}\left(y_{1}+y_{2}+y_{3}+y_{4}+\cdots+y_{j}\right) \in P^{j}$, whereas in quadratic expression, the variance with direction $\rho$ is as under:

$$
\sigma^{2} *(\rho)=\frac{1}{j} \sum_{l=1}^{j}\left[\rho^{C}\left(z_{l}-\hat{z}\right)\right]^{2}=\rho^{C} \Sigma \rho
$$

In preceding expression, mean of sample variance-covariance is denoted with $\Sigma$ that is presented as under:

$\Sigma_{\text {sample }}=\frac{1}{j} * \Sigma_{l=1}^{j}\left(z_{l}-\hat{z}\right)\left(z_{l}-\hat{z}\right)^{C}$ Equation 1

The covariance matrix, in the above equation-1, holds the characteristics of symmetrical, positive, and semi-definite. By the use of the above matrix, the computation of variance can be done in any direction.

\section{The constant correlation covariance matrix (overall mean)}

Elton and Gruber (1973) introduced the method of constant correlation to estimate the covariance matrix, backed with the assumption of variance of return for every asset category is a sample return. In contrast, the covariance of the same asset category is connected to the same coefficient of correlation. Due to this reason, the mean correlations coefficient is considered for the entire asset category. estimate covariance matrix using the constant correlation covariance matrix, claiming that the method performs well compared and is more appropriate than other estimators. Based on the said assumption, the average correlation coefficient is taken for entire asset categories.

As $\sigma_{s f}=\varphi_{s, i} \sigma_{s} \sigma_{f}$

So,

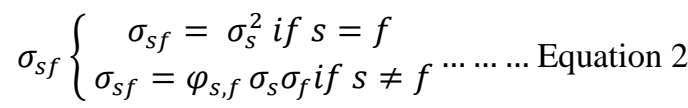

\section{The Single index variance-covariance model}

Sharpe (1963) constructs the single index method, backed by the assumption that returns for an asset category form a linear function for a market portfolio. It demonstrates that the returns on asset and market portfolios are linearly related. This linear interaction is significantly positive. Mathematically, this can be expressed as follows:

$$
R_{t u}=\mathrm{a}_{\mathrm{t}}+\mathrm{b}_{\mathrm{t}} \mathrm{x}_{\mathrm{t}}+\varepsilon_{\mathrm{t}}
$$


In preceding expression, $\mathrm{x}_{\mathrm{t}}$ denotes to the market portfolio which is correlated with its error term while $E *\left(\varepsilon_{t u} * \varepsilon_{h c}\right)=$ 0 . Where the variance $\left(\sigma^{2} *\left(\varepsilon_{t u}\right)=\vartheta_{t c}\right)$ within the asset category rests unaltered. Then, the matrix of covariance of $\left(\sigma_{t h}\right)$ can be formulated as follows:

$\sigma_{t h}=b \sigma^{2} * b^{2}+\partial$

In the preceding equation, $b$ denotes to the estimates of slope for the vector, $\sigma^{2}$ denotes to market variance whereas $\partial$ denotes to error term for the variance of the matrix. For single index covariance estimator can be written as:

$\sum_{[\text {single index }]}=\beta \sigma^{2} \hat{\beta}+\omega \ldots \ldots \ldots$ Equation 3

In the above equation, $\beta$ denotes to vector slope, $\sigma^{2}$ denotes to the sample market variance, whereas $\omega$ denotes to estimates of the matrix for the error term of the variance.

\section{Portfolio of Estimators}

The model of optimal weighted intensity by Ledoit and Wolf (2003) received criticism from Jagannathan and Ma (2003) later introduced the equally-weighted method of covariance estimation. This suggested framework for the estimation of variance-covariance was well-received by the community. This study estimates five equal-weighted (EW) portfolios, similar to the work of Liu and Lin (2010), Disatnik and Benninga (2007), and Jagannathan and Ma (2003).

\section{a. The portfolio based on sample \& diagonal matrix:}

Equation 4 below presents an equally weighted matrix of the sample and diagonal covariance where offdiagonal values are zero and variances for asset categories are diagonal items.

$\Sigma_{(\text {port-1 })}=\frac{1}{2} * \Sigma_{(\text {Sample Matrix })}+\frac{1}{2} * \Sigma_{(\text {Diagonal Matrix })} \ldots \ldots \ldots$ Equation 4

b. The portfolio based on sample \& single index matrix:

Equation 5 below is presenting an equally weighted matrix of sample and single index covariance estimator.

$\Sigma_{(\text {port }-2)}=\frac{1}{2} * \Sigma_{(\text {Sample Matrix })}+\frac{1}{2} * \Sigma_{(\text {Single Index Matrix })} \ldots \ldots \ldots$ Equation 5

c. The portfolio based on sample \& constant correlation covariance matrix:

Equation 6 below is presenting the matrix of sample \& constant correlation covariance (with overall mean).

$\Sigma_{(\text {port }-3)}=\frac{1}{2} * \Sigma_{(\text {Sample Matrix })}+\frac{1}{2} * \Sigma_{(\text {Overall Mean })} \ldots \ldots \ldots$ Equation 6

d. The portfolio is based on the sample, constant corr. \& single index matrix:

Equation 7 below presents a matrix of the sample, single index, and constant correlation covariance (with overall mean).

$\Sigma_{(\text {port }-4)}=\frac{1}{3} * \Sigma_{(\text {Sample Matrix })}+\frac{1}{3} * \Sigma_{(\text {Single Index Matrix })}+\frac{1}{3} * \Sigma_{(\text {overall Mean })} \ldots \ldots \ldots$ Equation 7

e. The portfolio based on the sample, single index \& overall mean matrix:

Equation 8 below presents the sample matrix, overall mean (mean of constant correlation), single index, and diagonal covariance estimator.

$\Sigma_{(\text {port }-5)}=\frac{1}{4} * \Sigma_{(\text {Sample Matrix })}+\frac{1}{4} * \Sigma_{(\text {Single Index Matrix })}+\frac{1}{4} * \Sigma_{(\text {Overall Mean })}+\frac{1}{4} *$

$\Sigma_{(\text {Diagonal Matrix })} \ldots \ldots \ldots$ E..... Equation 8

\section{Shrinkage variance-covariance matrix}

Ledoit and Wolf (2003) argue that the sample covariance and single index covariance method are error-prone. Both methods are greatly criticized in literature owing to their inherent problematic properties. The sample covariance method has an estimation problem, while the single index covariance matrix has a specification problem. These methods are inverse of each other as the single index method is the one-factor model, whereas the sample covariance method is the Zfactor model. Normally, a true estimating model will be an $n$-factor estimator: s.t. $Z>n>1$.

Stein (1956) presents the idea that an optimal point can be found using the weighted average for both estimators. This method guides toward shrinking the sample variance-covariance matrix toward a fixed diagonal target matrix. Chan et al. (1999), Bengtsson and Holst (2002), Ledoit and Wolf (2004) suggest through empirical evidence the usefulness of shrinkage estimation with a portfolio of estimators for the calculation of variance-covariance matrix. Jorion (1986) recommends the shrinkage method as it plays a major role in the portfolio selection process.

Suppose, $\tau$ and $\partial$ are sub-models of high and low dimension unrestricted parameters. The corresponding elements can be obtained using $C=\hat{\tau}$ and $D=\hat{\partial}$ from data. $C$ denotes to high variance due to its requirement of more fitted parameters compared to the $D$ as it is hypothetically biased. The estimator can be expressed as follows:

$\Sigma=\varnothing * \mathrm{D}+(1-\emptyset) * \mathrm{C} \ldots \ldots \ldots$ Equation 9 
In the previous equation, $C$ represents the sample covariance matrix, $D$ denotes to target matrix (highly organized estimator) whereas $\emptyset$ shows the weight of $D$, between $D$ and $C$ (in a convex linear fashion). The intensity of shrinkage $\emptyset$ ranges from zero to one.

The decision of the sample matrix and the shrinkage matrix depends on the value $\emptyset$. If the value is 1 , there is complete shrinkage (the obtained matrix is equal to target $D$ ); if the value is 0 means no-shrinkage, and thus, it reverts to the sample matrix. Now, the question arises, should the value $\emptyset$ be fixed or should it be ascertained using minimization of the following loss function:

$\mathrm{R}(\varnothing)=\mathrm{E}\left(\Sigma_{\mathrm{i}=1}^{\mathrm{p}}\left(\mathrm{m}_{\mathrm{i}}^{*}-\partial_{\mathrm{i}}\right)^{2}\right) \ldots \ldots \ldots$ Equation 10

Ledoit and Wolf (2003) proposed a method to ascertain optimal shrinkage intensity; this method aims at shrinking the sample variance-covariance matrix to a single index matrix. The basic idea is to minimize mean square error with no presupposition on distribution. As per the $1^{\text {st }}$ and $2^{\text {nd }}$ moments of $C$ and $D$, the formula based on squared error loss function is as follows:

$$
\begin{gathered}
R(\varnothing)=\sum_{i=1}^{p} \operatorname{var}\left(m_{i}^{*}\right)+\left[E *\left(\emptyset_{i}^{*}\right)-\tau_{i}\right]^{2} \\
=>\sum_{i=1}^{p} \operatorname{var} *\left(\varnothing t_{i}+(1-\emptyset) m_{i}\right)+\left[E *\left(\emptyset t_{i}+(1-\emptyset) m_{i}\right)-\tau_{i}\right]^{2} \\
=>\sum_{i=1}^{p} \emptyset^{2} \operatorname{var} *\left(g_{i}\right)+(1-\emptyset)^{2} \operatorname{var} *\left(\tau_{i}\right)+2 \emptyset(1-\emptyset) \operatorname{cov} *\left(m_{i}, g_{i}\right)+\left[\theta E\left(g_{i}-m_{i}\right)+\text { bias } *\left(m_{i}\right)\right]^{2}
\end{gathered}
$$

After minimization of the above function, w.r.t $\emptyset$;

$$
\emptyset^{*}=\frac{\sum_{i=1}^{p} v a r *\left(m_{i}\right)-\operatorname{cov} *\left(m_{i}^{\prime} g_{i}\right)-b i a s *\left(m_{i}\right) E *\left(g_{i}-m_{i}\right)}{\sum_{i=1}^{p} E *\left(g_{i}-m_{i}\right)^{2}}
$$

If $L$ in the preceding equation is an unbiased measure of $\tau$, The above equation can also be expressed in the following manner:

$\emptyset^{*}=\frac{\Sigma_{\mathrm{i}=1}^{\mathrm{p}} v \operatorname{var} *\left(\mathrm{~m}_{\mathrm{i}}\right)-\operatorname{cov} *\left(\mathrm{~m}_{\mathrm{i}^{\prime} \mathrm{g}_{\mathrm{i}}}\right)}{\Sigma_{\mathrm{i}=1}^{\mathrm{p}} \mathrm{E} *\left(\mathrm{~g}_{\mathrm{i}}-\mathrm{m}_{\mathrm{i}}\right)^{2}} \ldots \ldots \ldots$ Equation 11

The above equation 11 is used to calculate $(\varnothing)$ the optimal shrinkage intensity. Ledoit and Wolf (2004) provide the formula for the calculation of optimal shrinkage intensity and shrunk sample matrix of covariance to the constant matrix of correlational covariance. Literature shows that Bengtsson and Holst (2002) and Kwan (2011) shrink the sample covariance matrix toward S-factor the PCA and a diagonal matrix. Inconsistency with prior literature, this study uses three types of shrinkage techniques; shrinkage to a diagonal matrix, shrinkage technique to a single index, and shrinkage technique to constant correlation model.

\section{Evaluation parameters}

In line with previous research, this study uses two parameters for the evaluation of the covariance estimator. To check the pair-to-pair accuracy and ex-post accuracy of covariance estimators dataset is divided into two subsamples. As stated earlier, the first set of data ranges from 01/07/2006 to 30/06/2013, while the second data ranges from 01/07/2013 to $30 / 06 / 2020$. For evaluation, two different assessment parameters are considered. The first parameter is the root mean square error, and the second is the risk character of minimum variance portfolios. In the study, RMSE is estimated as under:

$=\sqrt{\frac{\mathrm{Q}(\mathrm{Q}-1)}{2} \sum_{\mathrm{f}=1}^{\mathrm{Q}} \times \sum_{\mathrm{t}=1, \mathrm{f} \neq \mathrm{l}}^{\mathrm{Q}}\left(\hat{\sigma}_{\mathrm{ft}}-\sigma_{\mathrm{ft}}\right)^{2}} \ldots \ldots \ldots$ Equation 12

In the above equation, $Q(Q-1) / 2$ represents pair-to-pare covariance estimators for an order of $\mathrm{Q} * \mathrm{Q}$ of the covariance matrix. The $\sigma_{f t}$ represents to real covariances and the $\hat{\sigma}_{f t}$ estimated values of between $f$ and $t$ covariances. A low value of RMSE is preferred over a low value in the pair-to-pair accuracy of the covariance estimator.

Consistent with the work of Saghir and Tirmizi (2020), Husnain et al. (2016), and Chan et al. (1999) the second evaluation parameter being applied in the study is the MVP method, which is estimated to see how effective estimators of covariances are in the selection of MVP. The MVP is later considered for the comparison of the results of estimators. An MVP is an exclusive portfolio, which basis on the covariance matrix but not on the return of asset category. A study using the first subsample calculates weights through MVP for every covariance estimator; later, these weights are considered in the estimation of note of the second subsample (out of sample returns). The series of return estimates for portfolio guides to the average MVP mean values and for risk behavior (Standard deviation of MVP). 
For MVP, the weight of $t$ risky asset is stated:

Minimum $_{\cdot w} w^{\varphi} * \Sigma w$, s.t. $w^{\varphi} e=1$

By the use of $\lambda$, the Lagrangian Multiplier, the above problem is restructured as follow:

$$
\text { Minimum }_{\cdot w} \partial=w^{\varphi} *\left[\Sigma w-2 \lambda *\left(w^{\varphi} e-1\right)\right]
$$

$\frac{\tau \partial}{\tau w}=>2 * \Sigma w-2 \lambda e=0$ (Under the rule of 1st order)

Solving for $w$, we get $w=\lambda *\left(\Sigma^{-1} e\right)$. Assume $h$ as a $P^{*} l$ vector. We write it as $h=1 / \lambda * w$, which can be stated as $h$ $==\Sigma^{-1}$ e. As we know, sum of weights equals to 1 (One), then $\mathrm{h}^{\varphi} \mathrm{e}=\frac{1}{\lambda} * \mathrm{w}^{\varphi} \mathrm{e}=\frac{1}{\lambda}$. So, the weight of any investment project for MVP is as under:

$\mathrm{w}_{(\mathrm{m} v \mathrm{p})}=\frac{\mathrm{h}}{\mathrm{h}^{\varphi} \mathrm{e}} \ldots \ldots \ldots$ Equation 13

\section{RESULTS AND DISCUSSION}

The results of RMSE for eleven variance-covariance estimators are presented in Table 2 . In accordance with the Liu \& Lin (2010) and Husnain et al. (2016) demonstrations pairwise values of variance-covariance matrices and corresponding out-of-sample results. A low value of RMSE is said to be better in comparison to other contending covariance estimators.

Table 2: RMSE results for variance-covariance estimators

\begin{tabular}{llr}
\hline Sr. & Variance-Covariance Estimators & Values \\
\hline & Traditional Methods & 0.01757 \\
\hline $\mathbf{1}$ & The sample var. covariance model (SC) & 0.01362 \\
\hline $\mathbf{2}$ & The constant correlation var. covariance model (CC) & 0.01370 \\
\hline \multicolumn{3}{l}{ Factor Model } \\
\hline $\mathbf{3}$ & The single index var. covariance model (SI) & 0.00879 \\
\hline \multicolumn{3}{l}{ Portfolio of Estimators } \\
\hline $\mathbf{4}$ & The portfolio based on sample \& diagonal matrix (P1) & 0.01411 \\
\hline $\mathbf{5}$ & The portfolio based on sample \& single index matrix (P2) & 0.01257 \\
\hline $\mathbf{6}$ & The portfolio based on sample \& constant correlation covar. matrix (P3) & 0.01193 \\
\hline $\mathbf{7}$ & The portfolio based on the sample, constant corr. \& single index matrix (P4) & 0.00895 \\
\hline $\mathbf{8}$ & The portfolio based on sample, single index \& overall mean matrix (P5) & 0.01749 \\
\hline & Shrinkage Approaches & 0.01419 \\
\hline $\mathbf{9}$ & The shrinkage aimed at diagonal point (SD) & 0.01040 \\
\hline $\mathbf{1 0}$ & The shrinkage aimed at single index point (SSI) & \\
\hline $\mathbf{1 1}$ & The shrinkage aimed at constant correlation point (SCC) \\
\hline
\end{tabular}

Table 2 shows that $\mathrm{P} 1$, the portfolio based on sample \& diagonal matrix estimator outperformed all other competing variance-covariance estimating methods. Results show that SC, the sample variance-covariance matrix performed worst among all. The results of P5, P4, P3 under the group of the portfolio of estimators, proposed by Jagannathan and Ma (2003) performed relatively good compared to the constant correlation var. covariance model (CC) and the single index variance-covariance model (SI) excluding P2, The portfolio based on sample \& single index matrix. Results show that the single index variance-covariance model (SI) performed slightly better than the portfolio based on the sample \& single index matrix (P2). However, the P2, the portfolio based on sample \& single index matrix, performed well compared to complex shrinkage approaches, i.e., the shrinkage aimed at the single index point (SSI) and the shrinkage aimed at the diagonal point (SD). Results also reveal that complex shrinkage approaches SSI and SD formulized by Ledoit \& Wolf $(2003,2004)$. Ledoit and Wolf (2004) performed worse than estimators of equally-weighted portfolios; P1, P5, P4, P3, and P2 respectively, suggested by Jagannathan and Ma (2003), except the shrinkage aimed at constant correlation point (SCC) which performed relatively better compared to P4, P3, and P2. Findings suggest that use of relatively tricky methods of variance-covariance estimation yield no extra benefit when evaluated against equally weighted methods of variance-covariance.

Table 3 presents standard deviation values of eleven variance-covariance for minimum variance portfolios (MVPs). Results show almost similar findings for both evaluation parameters, i.e., MVPs and RMSE, with some minor differences for MVPs.

Table 3: Standard deviation (Risk profile) for MVPS for alternative variance-covariances

\begin{tabular}{lll}
\hline Sr. & Variance-Covariance Estimators & Values \\
\hline & Traditional Methods & \\
\hline
\end{tabular}




\begin{tabular}{llr}
\hline $\mathbf{1}$ & The sample var. covariance model (SC) & 0.02063 \\
\hline $\mathbf{2}$ & The constant correlation var. covariance model (CC) & 0.02007 \\
\hline & Factor Model & 0.01966 \\
\hline $\mathbf{3}$ & The single index var. covariance model (SI) & 0.02007 \\
\hline & Portfolio of Estimators & 0.01983 \\
\hline $\mathbf{4}$ & The portfolio based on sample \& diagonal matrix (P1) & 0.02015 \\
\hline $\mathbf{5}$ & The portfolio based on sample \& single index matrix (P2) & 0.01983 \\
\hline $\mathbf{6}$ & The portfolio based on sample \& constant correlation covar. matrix (P3) & \\
\hline $\mathbf{7}$ & The portfolio based on sample, constant corr. \& single index matrix (P4) & 0.01975 \\
\hline $\mathbf{8}$ & The portfolio based on sample, single index \& overall mean matrix (P5) & 0.02063 \\
\hline $\mathbf{9}$ & Shrinkage Approaches & 0.02007 \\
\hline $\mathbf{1 0}$ & The shrinkage aimed at diagonal point (SD) & 0.01968 \\
\hline $\mathbf{1 1}$ & The shrinkage aimed at single index point (SSI) & \\
\hline
\end{tabular}

In line with preceding RMSE results, the shrinkage aimed at diagonal point (SD) and the sample var. covariance model (SC) again remain week performers in terms of standard deviation (SD). However, on the scale of standard deviation (SD), the single index variance-covariance model (SI) outperformed the competing estimators. As per the results of standard deviation, the portfolio based on sample \& diagonal matrix (P1) performed badly in comparison to the RMSE evaluation parameter. Similar to the results of RMSE, the shrinkage aimed at constant correlation point (SCC) again performed better on the measure of standard deviation (SD). Results also demonstrate that the other two complex methods of shrinkage estimators failed to deliver a good performance. Overall, the group of the portfolio of estimators, $\mathrm{P} 5, \mathrm{P} 2, \mathrm{P} 4$, and $\mathrm{P} 1$ performed well in comparison to the shrinkage aimed at single index point (SSI), the sample variance-covariance model (SC), and the shrinkage aimed at diagonal point (SD) except the portfolio based on sample \& constant correlation covariance matrix (P3) that did not do well. For comparison on related risk profiles, Table A1 presents results of average values, coherent with RMSE results and minimum variance portfolios (MVPs). Findings suggest that relatively more technical approaches of variance-covariance estimators provide no additional monetary advantage over the equally weighted portfolio of estimators.

For comparing different models of variance-covariance estimators, Table 4 presents the Sharpe ratio for resulting portfolios based on the minimum variance portfolio. Sharpe ratio reports risk-adjusted return using various inputs to MVP.

Table 4: Sharpe ratios for MVPs for alternative variance-covariance models

\begin{tabular}{llr}
\hline Sr. & Variance-Covariance Estimators & Values \\
\hline & Traditional Methods & 0.07190 \\
\hline $\mathbf{1}$ & The sample var. covariance model (SC) & 0.05497 \\
\hline $\mathbf{2}$ & The constant correlation var. covariance model (CC) & 0.07826 \\
\hline & Factor Model & \\
\hline $\mathbf{3}$ & The single index var. covariance model (SI) & 0.05368 \\
\hline & Portfolio of Estimators & 0.07464 \\
\hline $\mathbf{4}$ & The portfolio based on sample \& diagonal matrix (P1) & 0.06384 \\
\hline $\mathbf{5}$ & The portfolio based on sample \& single index matrix (P2) & 0.06867 \\
\hline $\mathbf{6}$ & The portfolio based on sample \& constant correlation covar. matrix (P3) & \\
\hline $\mathbf{7}$ & The portfolio based on sample, constant corr. \& single index matrix (P4) & 0.06085 \\
\hline $\mathbf{8}$ & The portfolio based on sample, single index \& overall mean matrix (P5) & \\
\hline & Shrinkage Approaches & 0.07165 \\
\hline $\mathbf{9}$ & The shrinkage aimed at diagonal point (SD) & 0.05747 \\
\hline $\mathbf{1 0}$ & The shrinkage aimed at single index point (SSI) & 0.07673
\end{tabular}

In Table 4, results of the Sharpe ratio show that the portfolio based on sample \& diagonal matrix (P1) outperformed all other competing methods of variance-covariance estimators. In contrast, the constant correlation variance-covariance model (CC) and complex approach to the shrinkage aimed at single index point (SSI) remain on the second and third best estimators. Results show that the single index var. covariance model (SI) performed worst among all estimators under the group of the factor model. Results also show that complex shrinkage models proposed by (Ledoit \& Wolf, 2003 , 2004) performed relatively worst in comparison to the equally weighted portfolio of estimators. Additionally, results also reveal that none of the single variance-covariance methods persistently outperformed the competing 
estimators. Findings confirm that technically advanced covariance methods do not benefit over equally weighted methods of the portfolio of estimators.

\section{CONCLUSION}

We evaluate the performance of eleven variance-covariance estimation methods by constructing four groups; traditional methods, factor models, equally-weighted portfolio of estimators, and shrinkage variance-covariance approaches in the presence of weekly data for the companies of the non-financial sector, listed at Pakistan stock exchange (PSX). The performance of variance-covariance estimators is judged for accuracy and effectiveness through RMSE and MVP two evaluation parameters.

From empirical results, it is clear that the sample and diagonal matrix's portfolio estimator outperformed all competing variance-covariance estimators while the sample covariance matrix performed worst as per RMSE parameters. For covariance estimators, both RMSE and MVP evaluation parameters yield different outcomes. The shrinkage to the diagonal-matrix technique and sample variance matrix performed poorly on the standard deviation scale, whereas the single index variance-covariance matrix outperformed all other contending estimators. Sharpe ratio results show that the sample and diagonal matrix's portfolio outperformed all other estimators, whereas complex shrinkage models of (Ledoit \& Wolf, 2003, 2004) performed worst compared to the group of weighted portfolios. Overall results show that the equally weighted variance-covariance portfolio of estimators introduced by Jagannathan and Ma (2003) performs considerably well compared to competing other covariance estimators. In line with previous research by Husnain et al. (2016), Nguyen (2018), the findings of the current study confirm that investors or asset management companies cannot receive extra financial gain from the use of more tricky methods of variance-covariance estimation compared to an equally weighted portfolio of estimators for the non-financial sector in the Pakistani equity market. In contrast to equally weighted portfolio estimators, investment executives are encouraged to proceed with caution when formulating an investment strategy incorporating the complex shrinkage variance-covariance approaches.

Future research may explore: a) other investment avenues such as money market instruments, gold futures contracts, commodity instruments, real properties such as land, crops, etc., and bond market using variance-covariance estimators for their effectiveness and accuracy. b) Studies may also use the data of financial sector companies listed at the Pakistan stock exchange, and c) datasets of other developing counties should also be considered to check the effectiveness of these estimators.

\section{AUTHOR'S CONTRIBUTION}

Ahsen Saghir and Syed Muhammad Ali Tirmizi introduced and presented the idea of this research. Ahsen Saghir, Ch Kamran Mahmood, and Naeem khan collected the required data and prepared the excel sheets for the final analysis. Syed Muhammad Ali Tirmizi proposed codes for data analysis. Ahsen Saghir and Nauman Iqbal Mirza performed data analyses. Ahsen Saghir and Naeem Khan complied and wrote the final draft.

\section{ACKNOWLEDGEMENT}

I would like to express my very great appreciation to Dr Syed Muhammad Ali Tirmizi for his continuous guidance and constructive suggestions for this research work.

\section{REFERENCES}

1. Bengtsson, C., \& Holst, J. (2002). On portfolio selection: Improved covariance matrix estimation for Swedish asset returns. Department of Economics, Luud University.

2. Best, M. J., \& Grauer, R. R. (1991). On the sensitivity of mean-variance-efficient portfolios to changes in asset means: some analytical and computational results. The Review of Financial Studies, 4(2), 315-342. https://doi.org/10.1093/rfs/4.2.315

3. Chan, L. K., Karceski, J., \& Lakonishok, J. (1999). On portfolio optimization: Forecasting covariances and choosing the risk model. The Review of Financial Studies, 12(5), 937-974.

4. Chopra, V., \& Ziemba, W. (1993). The Effect of Errors in Means, Variances, and Covariances on Optimal Portfolio Choice. The Journal of Portfolio Management, 19(2), 6. https://doi.org/10.3905/jpm.1993.409440

5. Chow, P. S., Cioffi, J. M., \& Bingham, J. A. (1995). A practical discrete multitone transceiver loading algorithm for data transmission over spectrally shaped channels. IEEE Transactions on communications, 43(2/3/4), 773-775. https://doi.org/10.1109/26.380108

6. DeMiguel, V., Garlappi, L., \& Uppal, R. (2007). Optimal Versus Naive Diversification: How Inefficient is the 1/N Portfolio Strategy? The Review of Financial Studies, 22(5), 1915-1953. https://doi.org/10.1093/rfs/hhm075

7. Disatnik, D. J., \& Benninga, S. (2007). Shrinking the covariance matrix. The Journal of Portfolio Management, 33(4), 55-63.

8. Elton, E. J., \& Gruber, M. J. (1973). Estimating the dependence structure of share prices-implications for portfolio selection. The Journal of Finance, 28(5), 1203-1232.

9. Husnain, M., Hassan, A., \& Lamarque, E. (2016). A Framework for Asset Allocation in Pakistani Equity Market: Simpler is Better. Pakistan Journal of Social Sciences (PJSS), 36(2), 881-893. https://bit.ly/3rcqd01 
10. Hwang, I., Xu, S., \& In, F. (2018). Naive versus optimal diversification: Tail risk and performance. European Journal of Operational Research, 265(1), 372-388. https://doi.org/10.1016/j.ejor.2017.07.066

11. Jagannathan, R., \& Ma, T. (2003). Risk Reduction in Large Portfolios: Why Imposing the Wrong Constraints Helps. The Journal of Finance, 58(4), 1651-1683. https://doi.org/10.3386/w8922

12. Jorion, P. (1986). Bayes-Stein estimation for portfolio analysis. Journal of Financial and Quantitative Analysis, 21(3), 279-292. https://doi.org/10.2307/2331042

13. King, B. F. (1966). Market and industry factors in stock price behavior. the Journal of Business, 39(1), 139190.

14. Konno, H., \& Yamazaki, H. (1991). Mean-absolute deviation portfolio optimization model and its applications to Tokyo stock market. Management Science, 37(5), 519-531.

15. Kwan, C. C. (2011). An introduction to shrinkage estimation of the covariance matrix: A pedagogic illustration. Spreadsheets in Education (eJSiE), 4(3), 6.

16. Ledoit, O., \& Wolf, M. (2003). Improved estimation of the covariance matrix of stock returns with an application to portfolio selection. Journal of Empirical Finance, 10(5), 603-621. https://doi.org/10.1016/s09275398(03)00007-0

17. Ledoit, O., \& Wolf, M. (2004). Honey, I shrunk the sample covariance matrix. The Journal of Portfolio Management, 30(4), 110-119. https://doi.org/10.2139/ssrn.433840

18. Levy, H., \& Markowitz, H. M. (1979). Approximating expected utility by a function of mean and variance. The American Economic Review, 69(3), 308-317.

19. Liu, L., \& Lin, H. (2010). Covariance estimation: do new methods outperform old ones? Journal of Economics and Finance, 34(2), 187-195.

20. Ly, W. (2019). Optimized Portfolios vs. the Naive-Diversification Strategy Universitetet i Agder; University of Agder].

21. Markowitz, H. (1952). Portfolio Selection. The Journal of Finance, 7(1), 77-91. https://doi.org/10.2307 /2975974

22. Michaud, R. O. (1989). The Markowitz optimization enigma: Is 'optimized'optimal? Financial Analysts Journal, 45(1), 31-42. https://doi.org/10.2469/faj.v45.n1.31

23. Nguyen, H. L. (2018). Out-of-sample testing on portfolio performance in the Asian equity market: Can optimized portfolio outperformed simpler strategy? [Bachelor's Thesis, Aalto University, School of Business, Mikkeli Campus]. http://urn.fi/URN:NBN:fi:aalto-201809105086

24. Pafka, S., \& Kondor, I. (2004). Estimated Correlation Matrices and Portfolio Optimization. Physica A: Statistical Mechanics and its Applications, 343, 623-634. https://doi.org/10.1016/j.physa.2004.05.079

25. Saghir, A., \& Tirmizi, M. A. (2020). An Empirical Assessment of Alternative Methods of Variance-Covariance Matrix. International Review of Management and Business Research, 09(04). https://doi.org/10.30543/94(2020)-33

26. Sharpe, W. F. (1963). A simplified model for portfolio analysis. Management Science, 9(2), 277-293.

27. Stein, C. (1956). Inadmissibility of the usual estimator for the mean of a multivariate normal distribution.

28. Tamiz, M., \& Jones, D. (1996). Goal programming and Pareto efficiency. Journal of Information and Optimization Sciences, 17(2), 291-307. https://doi.org/10.1080/02522667.1996.10699283

29. Tu, J., \& Zhou, G. (2011). Markowitz meets Talmud: A combination of sophisticated and naive diversification strategies. Journal of Financial Economics, 99(1), 204-215. https://doi.org/10.1016/j.jfineco.2010.08.013

30. Vasicek, O. A. (1973). A note on using cross-sectional information in Bayesian estimation of security betas. The Journal of Finance, 28(5), 1233-1239.

31. Zakamulin, V. (2017). Superiority of optimized portfolios to naive diversification: Fact or fiction? Finance Research Letters, 22, 122-128. https://doi.org/10.1016/j.frl.2016.12.007

\section{APPENDIX}

\section{Explanation of average of MVPs}

These study analyses covariance estimators through the MPVs. Estimates of sample and out-of-sample results of performance (returns) are estimated using the MPV weights for different covariance matrices. Table A1 presents the calculated average minimum variance portfolios (MVPs)

Table A1: Average MVPs of variance-covariance estimators

\begin{tabular}{llr}
\hline Sr. & Variance-Covariance Estimators & Values \\
\hline & Traditional Methods & \\
\hline $\mathbf{1}$ & The sample var. covariance model (SC) & 0.00185 \\
\hline $\mathbf{2}$ & The constant correlation var. covariance model (CC) & 0.00137 \\
\hline & Factor Model & 0.00193
\end{tabular}




\section{Portfolio of Estimators}

\begin{tabular}{lll}
\hline $\mathbf{4}$ & The portfolio based on sample \& diagonal matrix (P1) & 0.00137 \\
\hline $\mathbf{5}$ & The portfolio based on sample \& single index matrix (P2) & 0.00185 \\
\hline $\mathbf{6}$ & The portfolio based on sample \& constant correlation covar. matrix (P3) & 0.00161 \\
\hline $\mathbf{7}$ & The portfolio based on sample, constant corr. \& single index matrix (P4) & 0.00169 \\
\hline $\mathbf{8}$ & The portfolio based on sample, single index \& overall mean matrix (P5) & 0.00153 \\
\hline & Shrinkage Approaches & \\
\hline $\mathbf{9}$ & The shrinkage aimed at diagonal point (SD) & 0.00185 \\
\hline $\mathbf{1 0}$ & The shrinkage aimed at single index point (SSI) & 0.00145 \\
\hline $\mathbf{1 1}$ & The shrinkage aimed at constant correlation point (SCC) & 0.00185
\end{tabular}

